Bundesgesundheitsbl - Gesundheitsforsch Gesundheitsschutz 2004 • 47:352-362 DOI 10.1007/s00103-004-0810-y

Leitthema: Krankenhaushygiene

W. Witte $\cdot$ B. Strommenger $\cdot$ I. Klare $\cdot$ G. Werner

Bereich Wernigerode, Robert Koch-Institut, Wernigerode

\title{
Bakterielle Erreger von Krankenhausinfektionen mit besonderen Resistenzen und Multiresistenzen
}

\section{Teil I: Diagnostik und Typisierung}

\section{Natürliche und erworbene Resistenz gegen Antibiotika}

Für fast jede der therapeutisch gegen bakterielle Infektionserreger eingesetzten Antibiotikagruppen gibt es mehrere Einzelpräparate, die in der klinisch-bakteriologischen Routinediagnostik in der Empfindlichkeitsprüfung nicht erfasst werden können. Deshalb werden bei Kenntnis der Resistenzmechanismen und der daraus abzuleitenden Kreuzresistenzen bestimmte Testpräparate geprüft und das diesbezügliche Ergebnis im Sinne der Kreuzresistenz interpretiert. Diese Testpräparate entsprechen den Substanzen, die auch in den Ausführungen zu $§ 23$ des Infektionsschutzgesetzes (IfSG) aufgeführt sind. Aufgrund der suboptimalen In-vitroExpression verschiedener Resistenzmechanismen ist es erforderlich, neben den Routinemethoden (Agardiffusionstest, Mikrobouillon-MHK) ergänzende Tests einzusetzen, vorzugsweise durch Nachweis der Resistenzgene. Das Auftreten und besonders die Verbreitung antibiotikaresistenter Erreger von Krankenhausinfektionen können durch Erregertypisierung verfolgt werden. Bei der Auswahl der Typisierverfahren ist es erforderlich, Arbeitsaufwand, Diskriminierungsfähigkeit und Reproduzierbarkeit einzuschätzen. Zukünftig wird die Anwendung der Mikroarray-Technologie eine schnelle komplexe Diagnostik ermöglichen, die neben den Spezies-Eigenschaften und den Resistenzdeterminanten auch Virulenzassoziierte Gene einschließt.
Die bisher therapeutisch eingesetzten antibakteriellen Chemotherapeutika hemmen Einzelschritte der in Abb. 1 gezeigten komplexen molekularen Vorgänge in der Bakterienzelle. Sind diese Zielstrukturen den Antibiotika von vornherein aufgrund der natürlichen Eigenschaften bestimmter Bakterienspezies nicht zugänglich, dann besteht eine natürliche Resistenz, d. h., diese Bakterien liegen nicht im Wirkungsspektrum eines Antibiotikums. Demgegenüber steht die erworbene Resistenz, die erst im Zuge des Selektionsdruckes durch den Antibiotikaeinsatz auftritt. Bakterien haben eine Reihe verschiedener molekularer Resistenzmechanismen entwickelt, die in Übersicht 1 zusammengefasst sind (Übersicht bei [1]).

\section{Auswahl von Antibiotika für die Resistenzbestimmung}

Die Antibiotika-Resistenzbestimmung hat 2 wesentliche Ziele: Sie dient einerseits der Absicherung der antibakteriellen Chemotherapie, soll aber auch rechtzeitig über das Auftreten und die Verbreitung von Resistenzen informieren. Gegenwärtig sind in Krankenhäusern meist 30-40 verschiedene Antibiotika gelistet. Aus Kapazitäts- und Kostengründen ist es aber unmöglich, für jede dieser Substanzen eine Resistenzbestimmung durchzuführen. Die Bestimmung

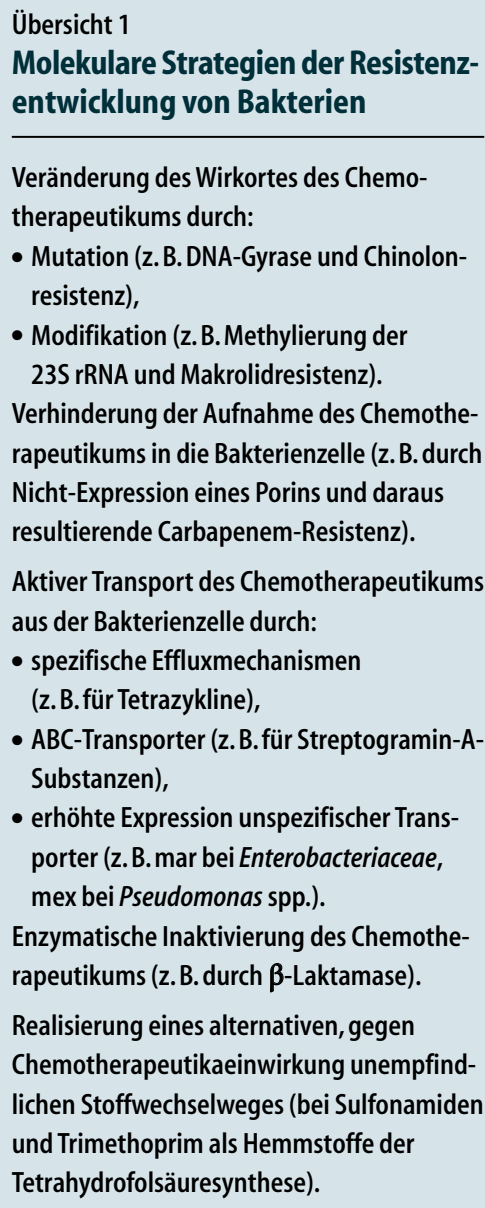

Aktiver Transport des Chemotherapeutikums aus der Bakterienzelle durch:

- spezifische Effluxmechanismen

(z. B. für Tetrazykline),

- ABC-Transporter (z. B. für Streptogramin-ASubstanzen),

- erhöhte Expression unspezifischer Transporter (z. B. mar bei Enterobacteriaceae, mex bei Pseudomonas spp.).

Enzymatische Inaktivierung des Chemotherapeutikums (z. B. durch $\beta$-Laktamase).

Realisierung eines alternativen, gegen Chemotherapeutikaeinwirkung unempfindlichen Stoffwechselweges (bei Sulfonamiden und Trimethoprim als Hemmstoffe der Tetrahydrofolsäuresynthese).

(C) Springer-Verlag 2004

Prof.Dr.W.Witte

Bereich Wernigerode, Robert Koch-Institut, Burgstraße 37,38855 Wernigerode

E-Mail:wittew@rki.de 
W.Witte $\cdot$ B. Strommenger $\cdot$ I. Klare G.Werner

\section{Antibiotic-resistant nosocomial pathogens. Part l: diagnostic and typing methods}

\section{Abstract}

For use in human chemotherapy, there are several different substances for nearly each substance group available which cannot all be checked in routine susceptibility testing. If the bacterial resistance mechanisms and cross-resistance conferred by them are known, particular test substances can be selected and the results are interpreted on the basis of cross-resistance. Test substances correspond to those mentioned in guidelines for § 23 IfSG (German law on protection against infection). Due to suboptimal in vitro expression of different resistance mechanisms, it is necessary to perform additional tests besides routine agar-diffusion or microbroth MIC assays. These are preferentially tests for molecular demonstration of resistance genes. Emergence and spread of antibiotic-resistant nosocomial pathogens can be traced by typing. When selecting a typing method, it is important to assess work load, discriminatory power, and reproducibility. In future the availability of microarray technology will enable routine laboratories to demonstrate particular virulence-associated traits.

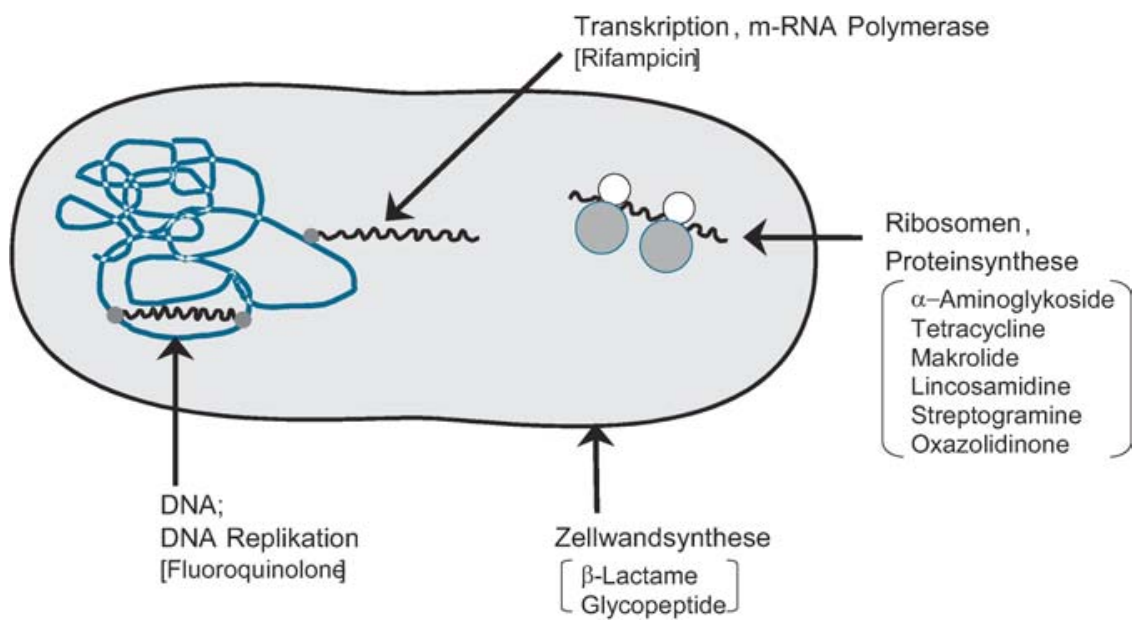

Abb. $1 \Delta$ Zielstrukturen für Antibiotika

dient der Ermittlung der erworbenen Resistenz, natürliche Resistenzen werden aus der Speziesbestimmung abgeleitet. Die Mehrheit der heute verfügbaren Antibiotika lässt sich einer begrenzten Zahl von Substanzklassen zuordnen, die (mit Ausnahme der Oxazolidinone) zwischen Mitte der 3oer-Jahre bis zur Mitte der 6oer-Jahre des vorigen Jahrhunderts entdeckt bzw. entwickelt wurden. Ziel der Weiterentwicklung der Präparate innerhalb einer Substanzklasse war die Verbesserung der Pharmakokinetik und die Verringerung der Toxizität, aber auch das Überwinden von Resistenzmechanismen der Bakterienzelle. Letzteres wird an der Entwicklung der $\beta$-Laktamantibiotika besonders deutlich (Abb. 2).

Ein bestimmter Resistenzmechanismus führt häufig zu einer Unempfindlichkeit des Bakteriums gegen alle Präparate einer Substanzklasse. Zum Beispiel bedingen durch Staphylokokken gebildete $\beta$-Laktamasen eine Resistenz gegen alle Substanzen, die gegen diese $\beta$-Laktamase empfindlich sind. Es liegt dann eine Kreuzresistenz vor. In diesen Fällen ist es ausreichend und sinnvoll, eine bestimmte Substanz als Testpräparat zu prüfen und das Ergebnis aufgrund der bekannten Kreuzresistenzen auf andere Präparate der gleichen oder bezüglich ihrer Wirkungsweise (nicht nur unbedingt bezüglich der chemischen Struktur) verwandter Substanzen zu übertragen.

Bei bestimmten Erregerspezies können allerdings im Hinblick auf die Präparate einer Substanzgruppe verschiedene Resistenzmechanismen mit einem unterschiedlichen Substratspek- trum verbreitet sein (z. B. Aminoglykosidresistenz bei Pseudomonas spp.). Bei Resistenzbestimmungen mittels automatisierter Expertensysteme wird diese Tatsache bereits berücksichtigt und mit dem Befundausdruck übermittelt. Allerdings darf „Expertensystemen“ nicht blind vertraut werden. Eine Plausibilitätskontrolle sollte immer erfolgen, da z. B. neueste Erkenntnisse noch nicht in die Software eingespeist sein können oder die Komplexität der Resistenzmechanismen noch nicht vollständig erfasst wurde.

In der klinisch-bakteriologischen Routine ist es zweckmäßig, bezüglich der Testreihen von Präparaten für die Resistenzbestimmung zwischen grampositiven und gramnegativen Bakterien zu unterscheiden, bei letzteren ggf. auch noch zwischen Enterobacteriaceae und Pseudomonaden. Bei grampositiven Bakterien - insbesondere bei den Staphylokokken - sind für die verschiedenen Substanzgruppen vergleichsweise wenig Resistenzgene und dementsprechend auch Resistenzmechanismen verbreitet. Die Festlegung von Testsubstanzen und die Interpretation der Ergebnisse bezüglich der Kreuzresistenzen sind daher unproblematisch (Tabelle 1 und 2, Übersicht bei [2]).

Bei gramnegativen Bakterien kann einer definierten Antibiotikaresistenz jedoch eine erheblich größere Vielfalt an Resistenzgenen und damit auch Resistenzmechanismen zugrunde liegen. Dennoch ist es in vielen Fällen möglich, aufgrund des Substratspektrums und der Kreuzresistenzen bestimmte Testpräparate auszuwählen und das Ergeb- 


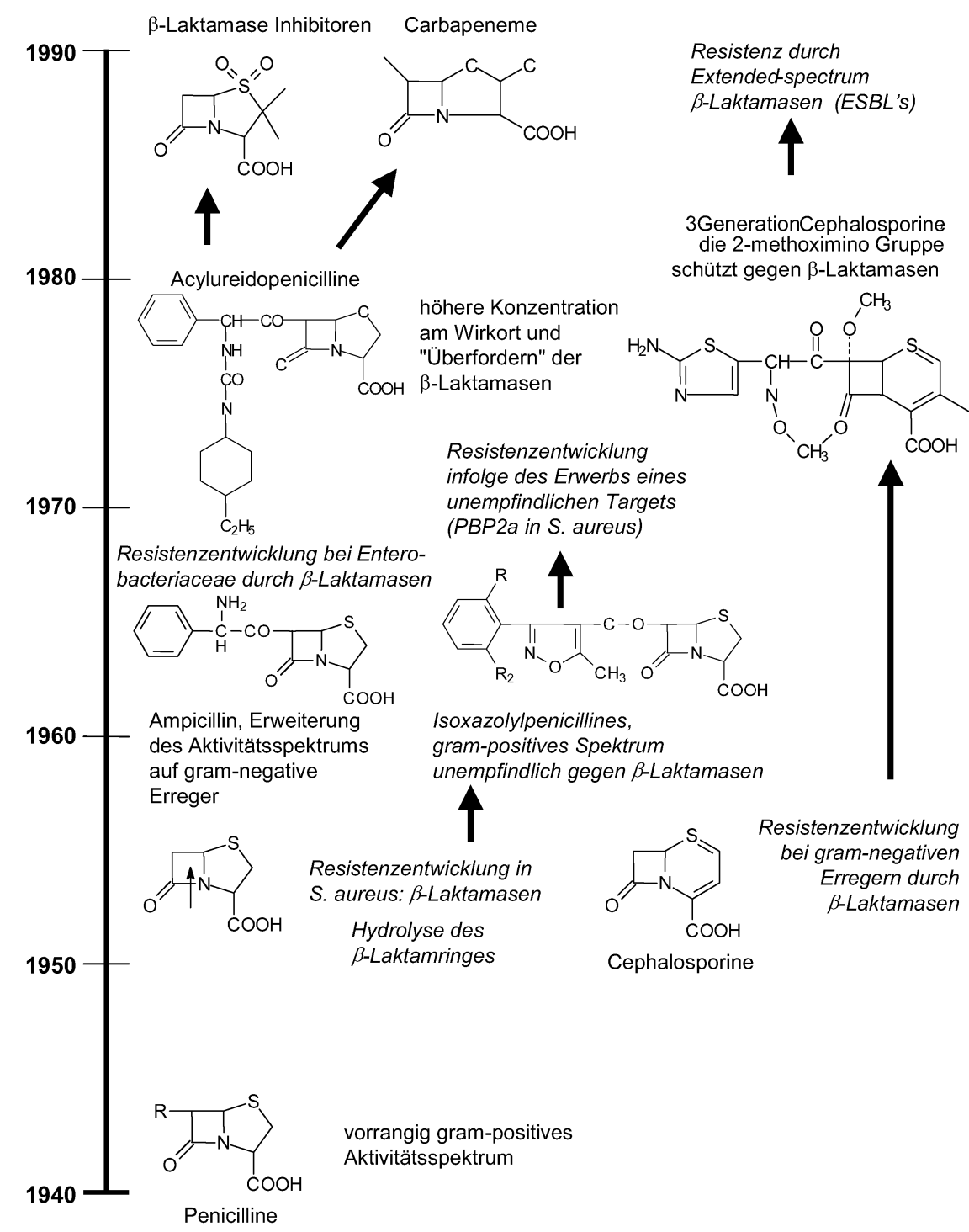

Abb. $2 \boldsymbol{\Delta}$ Weiterentwicklung von $\boldsymbol{\beta}$-Laktamantibiotika im Zuge der Resistenzentwicklung bakterieller Infektionserreger

nis der Resistenzbestimmung entsprechend zu interpretieren (Tabelle 3 für Enterobacteriaceae, Tabelle 4 für Pseudomonas spp., Übersicht zur Aminoglykosidresistenz bei $[3,4])$.

Von aktueller Bedeutung für die Resistenzbestimmung bei Enterobacteriaceae ist das richtige Erkennen von Resistenzen gegen Cephalosporine der 3. und 4. Generation aufgrund von $\beta$-Laktamasen mit erweitertem Substratspektrum (ESBL). Diese können aus unterschiedlichen plasmidkodierten $\beta$-Laktamasen durch ein oder mehrere Aminosäurenaustauschmutationen hervorgegangen sein (Übersicht bei [5]). Diese $\beta$-Laktamasen gehören zu den Klassen A und D. In Abhängigkeit von ihrer evolutionären Herkunft zeigen sie ein unterschiedli- ches Substratspektrum (Tabelle 5). Allerdings werden durch den Test für Cefotaxim und Ceftazidim fast alle ESBL gut erfasst. Da plasmidkodierte $\beta$-Laktamasen fast immer durch $\beta$-Laktamaseinhibitoren gehemmt werden, sollte parallel der Test mit Cefpodoxim (allein und in Kombination mit Clavulansäure) erfolgen.

Das seit Januar 2001 in Deutschland geltende Infektionsschutzgesetz (IfSG) verpflichtet Krankenhäuser dazu, für die wichtigsten Erreger nosokomialer Infektionen eine Resistenzstatistik zu führen. Dabei sollen aus klinischer und epidemiologischer Sicht wichtige Resistenzeigenschaften erfasst werden. Eine entsprechende Übersicht gibt Tabelle 6 .

\section{Methoden der Resistenz- bestimmung im Überblick}

\section{Phänotypische Verfahren}

Am weitesten verbreitet ist immer noch der Agardiffusionstest, für dessen Durchführung in Deutschland der DINStandard 58940 [6] gilt. Neben dieser Empfehlung wird in Deutschland auch der NCCLS-Standard [7] eingesetzt. Es ist dabei grundsätzlich zu beachten, dass diese Standards unterschiedliche Inokulumdichten und unterschiedliche Blättchenbeladungen verwenden und somit auch unterschiedliche Ablesekriterien für die Bewertungsstufen als empfindlich, intermediär und resistent festlegen. Die einzelnen Komponenten (z. B. Inokulum nach DIN, Blättchen nach NCCLS, Ablesung nach DIN) sind nicht austauschbar! Der Agardiffusionstest ist aufgrund der Komplexität des Verfahrens deutlich höher mit Fehlern belastet als die Referenzmethode, d. h. die Bestimmung minimaler Hemmkonzentrationen (in der Praxis mittels Mikrobouillonverdünnungstest). Die Bestimmung minimaler Hemmkonzentrationen mittels E-Test (einem Diffusionstest unter Verwendung von MHK-Teststreifen) führt zu Ergebnissen, die eine hohe Übereinstimmung mit dem Mikrobouillonverdünnungstest zeigen.

Die Ergebnisse phänotypischer Resistenzverfahren können generell durch äußere Einflüsse auf die Expression der Gene, die für die Resistenz kodieren, beeinträchtigt werden. Faktoren wie Temperatur, pH-Wert, die Konzentration von Salzen, die verwendeten Nährmedien und die Einstellung des Inokulums beeinflussen die Expression und können daher zu fehlerhaften Ergebnissen führen. $\mathrm{Zu}$ den phänotypischen Bestätigungsverfahren für Resistenzeigenschaften, die durch den Agardiffusionstest und die Bestimmung minimaler Hemmkonzentrationen nicht immer verlässlich erfasst werden, zählen:

Der Nachweis der Oxacillinresistenz bei MRSA (Methicillin-resistente Stapylococcus aureus), d. h. die Verwendung des Agar-Screening-Tests nach NCCLS [7] oder eines BouillonScreening-Verfahrens [8] bzw. eines Latex-Agglutinationstests zum Nachweis des Penicillinbindeproteins PBP2a [9], 
Tabelle 1

Auswahl der Testpräparate für die phänotypische Resistenzbestimmung bei Staphylokokken

\begin{tabular}{|c|c|c|}
\hline Testsubstanz & Resistenzmechanismus und Resistenzgene & Kreuzresistenzen \\
\hline Gentamicin & Aminoglycosid-modifizierendes Enyzm ( $a p h 2^{\prime \prime}$ - $\left.a a c \sigma^{\prime} \mathrm{Gen}\right)$ & Potenziell auch gegen Amikacin, Netilmicin \\
\hline Erythromycin & $\begin{array}{l}\text { Methylierung der } 235 \text { r-RNA in Position } 2058 \\
\text { (ermA, ermB, ermC) }\end{array}$ & Alle Makrolide \\
\hline Linkomycin & Methylierung der 23S r-RNA in Position 2058 & $\begin{array}{l}\text { Bei konstitutiver Ausprägung alle Linkosamidine, } \\
\text { Streptogramin-B-Substanzen }\end{array}$ \\
\hline $\begin{array}{l}\text { Quinupristin/ } \\
\text { Dalfopristin }\end{array}$ & $\begin{array}{l}\text { Methylierung der } 23 S \text { r-RNA in Pos. } 2058 \text { (ermB) } \\
\text { für Quinupristin } \\
\text { 0-Acetylierung für Dalfopristin (vatA, vatB) }\end{array}$ & $\begin{array}{l}\text { Gegen andere Streptogramin-A/B- } \\
\text { Kombinationen }\end{array}$ \\
\hline Ciprofloxacin & Mutation Ser80 des grlA-Gens (Topoisomerase IV) & $\begin{array}{l}\text { Alle Fluorchinolone Gr. II, potenziell } \\
\text { alle Fluorchinolone }\end{array}$ \\
\hline Moxifloxacin & Zusätzlich Mutation in Ser83 des gyrA-Gens (DNA-Gyrase) & Alle Fluorchinolone \\
\hline $\begin{array}{l}\text { Trimethoprim/ } \\
\text { Sulfamethoxazol }\end{array}$ & Trimethoprim-unempfindliche Dihydrofolatreduktase $\mathrm{a}^{\mathrm{a}}$ & Alle Sulfonamide \\
\hline Rifampicin & $\begin{array}{l}\text { Punktmutation in der } \beta \text {-Untereinheit } \\
\text { der } m \text {-RNA-Polymerase }\end{array}$ & Andere Rifamycine \\
\hline $\begin{array}{l}\text { Fusidinsäure- } \\
\text { Natrium }\end{array}$ & Resistenzmutation, Efflux & \\
\hline $\begin{array}{l}\text { Vancomycin/ } \\
\text { Teicoplanin }\end{array}$ & $\begin{array}{l}\text { Dickere Zellwand mit unvollständiger Quervernet- } \\
\text { zung der Peptidoglykanstränge (trapping effect) }\end{array}$ & \\
\hline Linezolid & $\begin{array}{l}\text { Punktmutation(en) in der Domäne V der zentralen } \\
\text { Schleife der } 235 \text { r-RNA }\end{array}$ & \\
\hline
\end{tabular}

asulfonamid Dihydropteroatsynthase.

Tabelle 2

Auswahl von Testpräparaten für die phänotypische Resistenzbestimmung bei Enterokokken

\begin{tabular}{|c|c|c|}
\hline Testsubstanz & $\begin{array}{l}\text { Resistenzmechanismus } \\
\text { und Resistenzgene }\end{array}$ & Kreuzresistenzen \\
\hline Ampicillin & $\begin{array}{l}\text { E. faecalis: } \beta \text {-Laktamase } \\
\text { E. faecium: verändertes Penicillinbindendes Protein PBP5 }\end{array}$ & $\begin{array}{l}\text { Alle } \beta \text {-Laktamase-empfindlichen Penicilline } \\
\text { Mehr oder weniger alle } \beta \text {-Laktamantibiotika }\end{array}$ \\
\hline Vancomycin & $\begin{array}{l}\text { Targetmodifikation an dem } \\
\text { N-Azetylmuraminsäure-Pentapeptid }\end{array}$ & Teilweise Teicoplanin \\
\hline Teicoplanin & $\begin{array}{l}\text { Targetmodifikation an dem } \\
\text { N-Azetylmuraminsäure-Pentapeptid }\end{array}$ & Vancomycin \\
\hline Gentamicin & Modifizierendes Enzym (aph2"-aac6) & Amikacin, Netilmicin \\
\hline Streptomycin & Modifizierendes Enyzm (aad6) & - \\
\hline Erythromycin & Methylierung der 23S r-RNA in Pos. 2058 (ermB) & Streptogramin B bei konstanter Expression \\
\hline $\begin{array}{l}\text { Quinupristin/ } \\
\text { Dalfopristin }\end{array}$ & $\begin{array}{l}\text { E. faecium: Methylierung der 23S r-RNA } \\
\text { in Pos. } 2058 \text { (ermB) für Quinupristin } \\
\text { 0-Acetylierung für Dalfopristin } \\
\text { Bei E. faecalis natürliche Resistenz }\end{array}$ & Andere Streptogramin-Kombinationen \\
\hline Linezolid & $\begin{array}{l}\text { Mutation in der Domäne V der zentralen } \\
\text { Schleife der 23S rRNA }\end{array}$ & \\
\hline
\end{tabular}


Tabelle 3

Auswahl von Testpräparaten für die phänotypische Resistenzbestimmung bei Enterobacteriaceae

\begin{tabular}{|c|c|c|}
\hline Testsubstanz & $\begin{array}{l}\text { Resistenzmechanismus } \\
\text { und Resistenzgene }\end{array}$ & Kreuzresistenzen \\
\hline Aminopenicilline & $\beta$-Laktamasen & $\begin{array}{l}\text { Ampicillin, Amoxycillin, } \\
\text { ggf. Acylureidopenicilline }\end{array}$ \\
\hline $\begin{array}{l}\text { Ampicillin/Sulbactam, } \\
\text { Amoxycillin (Clavulansäure) }\end{array}$ & $\begin{array}{l}\text { Bei Resistenz aufgrund einer } \\
\text { Überproduktion von plasmid- } \\
\text { kodierten } \beta \text {-Laktamasen oder } \\
\text { ampC- } \beta \text {-Laktamasen }\end{array}$ & $\begin{array}{l}\text { Resistenz gegen Inhibitor- } \\
\text { geschützte Aminopenicilline }\end{array}$ \\
\hline Piperacillin (Mezlocillin) & $\begin{array}{l}\text { Exprimierte ampC- } \beta \text {-Laktamasen, } \\
\text { plasmiddeterminierte, } \\
\text { durch Inhibitor hemmbare } \\
\beta \text {-Laktamasen }\end{array}$ & $\begin{array}{l}\text { Potenziell gegen alle Amino- } \\
\text { und Acylureidopenicilline }\end{array}$ \\
\hline $\begin{array}{l}\text { Piperacillin/Tazobactam } \\
\text { (Piperacillin/Sulbactam) }\end{array}$ & $\begin{array}{l}\text { Ursprünglich durch Inhibitor- } \\
\text { hemmbare } \beta \text {-Laktamase } \\
\text { (plasmidkodiert) mit Mutation zur } \\
\text { Inhibitorresistenz; exprimierte } \\
\text { ampC- } \beta \text {-Laktamasen }\end{array}$ & Inhibitorgeschützte Penicilline \\
\hline Cefuroxim oder Cefotiam & $\beta$-Laktamasen & $\begin{array}{l}\text { Andere Gruppe-II-Cephalos- } \\
\text { porine, alle Penicilline }\end{array}$ \\
\hline $\begin{array}{l}\text { Cefotaxim, Ceftazidim, } \\
\text { parallel mit Clavulansäure }\end{array}$ & $\begin{array}{l}\text { ESBL exprimierte } \\
\text { ampC- } \beta \text {-Laktamasen }\end{array}$ & $\begin{array}{l}\text { Potenziell alle Cephalosporine } \\
\text { und Penicilline }\end{array}$ \\
\hline $\begin{array}{l}\text { Cefpodoxim,Cefpodoxim/ } \\
\text { Clavulansäure }\end{array}$ & $\begin{array}{l}\text { Test auf plasmiddeterminierte } \\
\text { ESBL, die durch Clavulansäure } \\
\text { ESBL, hemmbar sind }\end{array}$ & $\begin{array}{l}\text { Potenziell alle Cephalosporine } \\
\text { und Penicilline }\end{array}$ \\
\hline Imipenem & Fehlende Expression des OMP D & Meropenem \\
\hline Trimethoprim & Resistente Dihydrofolatreduktase & \\
\hline Gentamicin & $\begin{array}{l}\text { Verschiedene Aminoglykosid- } \\
\text { modizierende Enzyme [(aac(3) } \\
\text { bis X; ant }\left(2^{\prime \prime}\right) \text { I] }\end{array}$ & $\begin{array}{l}\text { Teilweise Tobramycin, } \\
\text { Netilmicin, Amikacin }\end{array}$ \\
\hline Amikacin & $\begin{array}{l}\text { Aminoglykosidacetyltransferase } \\
\operatorname{aac}\left(6^{\prime}\right) \text { I } \\
\text { Aminoglykosidphosphoryltrans- } \\
\text { ferase aph }\left(3^{\prime}\right) \mathrm{VI}\end{array}$ & $\begin{array}{l}\text { Gentamicin, Netilmicin, } \\
\text { Tobramycin }\end{array}$ \\
\hline
\end{tabular}

ESBL Extended Spectrum $\beta$-Laktamase.
Laktamasen mit erweitertem Spektrum (ESBL) zu. Inzwischen sind für die Methicillinresistenz Testkits verfügbar, die auf der Detektion der PCR-Produkte durch reverse Hybridisierung beruhen (reverser Blot, d. h. Hybridisierung des PCR-Produkts gegen eine spezifische Capture-Probe im Makroarray [10, 11]). Zum gleichzeitigen Nachweis mehrerer Resistenzgene wurden Multiplex-PCRVerfahren entwickelt (für Staphylokokken [12], für AmpC- $\beta$-Laktamasen [13]). Abbildung 3 zeigt dies für Staphylokokken. Die verschiedenen PCR-Produkte werden dabei mittels Gelelektrophorese oder Kapillarelektrophorese aufgetrennt. Die Zuordnung der einzelnen Banden zu einem gesuchten Resistenzgen erfolgt jedoch zunächst nur über die Größe des PCR-Produktes. Dieses Vorgehen birgt vor allem bei Multiplexsystemen die Gefahr falsch positiver Ergebnisse aufgrund des Vorliegens unspezifischer PCR-Produkte. Eine Kombination von Multiplex-PCR und reversem Blot verbessert die Spezifität der Detektion der Zielsequenz signifikant. Zu diesem Zweck werden statt der membranbasierten Hybridisierungssysteme zunehmend Mikrochips (Mikroarrays) eingesetzt. Auf der Glasoberfläche dieser Chips können spezifische Captureproben für Tausende von Zielgenen aufgebracht werden. Diese Systeme detektieren sowohl erworbene Resistenzgene $[14,15]$ als auch die Mutationen, die zu Resistenzen führen [für Staphylokokken z. B. Mutationen im Gen für die DNA-Topoisomerase ( $g r l \mathrm{~A})$, die zur Chinolonresistenz führen]. Die fraglichen DNA-Abschnitte werden zunächst mittels PCR präamplifiziert, mit fluoreszierenden Farbstoffen markiert und dann auf einem Microarray hybridisiert. Abbildung 4 zeigt die Ergebnisse solcher Hybridisierungen.

Für die besonders schnelle Detektion einzelner Resistenzgene in einer Vielzahl von klinischen Proben werden heute Real-time-PCR-Systeme eingesetzt: Durch die Verwendung sequenzspezifischer, fluoreszensmarkierter Reportermoleküle und die Online-Erfassung der erzeugten Fluoreszenzsignale lassen sich hier Resistenzgene und -mutationen besonders schnell und spezifisch detektieren. Allerdings ist die Möglichkeit zur simultanen Erfassung verschiedener Gene durch das beschränkte Angebot an Repoterfarbstoffen limitiert. 


\begin{tabular}{|c|c|c|}
\hline Testsubstanz & Resistenzmechanismus & Kreuzresistenzen \\
\hline Piperacillin & Chromosomale $\beta$-Laktamase, exprimiert & Azlocillin \\
\hline Ceftazidim & Chromosomale $\beta$-Laktamase, exprimiert & Z.T. für Cefsulodin \\
\hline Ciprofloxacin & Mutation in Ser84 von gyrA DNA-Gyrase & $\begin{array}{l}\text { Andere Fluorchinolone } \\
\text { der Gruppe II }\end{array}$ \\
\hline Imipenem & $\begin{array}{l}\text { Fehlendes } 49 \text { kD OMP+chromosomale } \beta \text {-Laktamase } \\
\text { exprimiert; Metallo- } \beta \text {-Laktamase, plasmidkodiert }\end{array}$ & Meropenem \\
\hline Gentamicin & $\begin{array}{l}\text { Aminoglykosidacetyltransferase } a a c(3) \text { I } \\
\text { Aminoglykosidacetyltransferase } a a c(3) \text { III } \\
\text { Aminoglykosidacetyltransferase } a a c\left(6^{\prime}\right) \text { II }\end{array}$ & $\begin{array}{l}\text { Sisomycin } \\
\text { Tobramycin } \\
\text { Tobramycin, Netilmicin }\end{array}$ \\
\hline Amikacin & Aminoglykosidnukleotidyltransferase ant( $\left.4^{\prime}\right)$ II & Tobramycin \\
\hline
\end{tabular}

Tabelle 5

Hydrolyse von ausgewählten Substanzen durch $\beta$-Laktamasen mit erweitertem Substratspektrum (Extended Spectrum $\beta$-Laktamasen, ESBL, Übersicht bei [5])

\begin{tabular}{lllllc} 
Enzyme & $\begin{array}{l}\text { Aminosäure- } \\
\text { substitution(en) }\end{array}$ & \multicolumn{3}{l}{ Relative Hydrolyserate } \\
\cline { 3 - 6 } & & Benzylpenicillin & Cephaloridin & Cefotaxim & Ceftazidim \\
\hline TEM-1 & & 100 & 140 & 0,07 & 0,01 \\
TEM-2 & Q39K & 100 & 120 & 0,08 & $<0,01$ \\
TEM-3 & Q39 K, E104 K, G238S & 100 & 120 & 170 & 8,3 \\
TEM-5 & R164S, A237T, E240K & 100 & 300 & 29 & 100 \\
TEM-7 & Q39 K, R164S & 100 & 120 & 1,9 & 1,7 \\
TEM-10 & R164S, E240K & 100 & 77 & 1,6 & 68 \\
TEM-12 & R164S & 100 & 57 & 2,4 & 3,8 \\
TEM-20 & G328S & 100 & 150 & 250 & $<1$ \\
TEM-26 & E104 K, R164S & 100 & 120 & 7,5 & 170 \\
SHV-1 & & 100 & 120 & 7,5 & 170 \\
SHV-2 & G238S & 100 & 48 & 0,18 & 0,02 \\
SHV-4 & R205L, G238S, E240K & 100 & 5,6 & 1,1 & 0,65 \\
SHV-7 & I8F, R43S, G238S, E240K & 100 & 91 & 30 & 13 \\
& & & & &
\end{tabular}

Für S. aureus wurden z. B. Systeme zur Speziesidentifikation und zum Nachweis des mecA-Gens entwickelt $[16,17]$.

\section{Molekulare Typisierung von Erregern nosokomialer Infektionen}

Die Typisierung im Rahmen epidemiologischer Untersuchungen hat das Ziel, den Epidemiestamm (Klon) durch bestimmte Merkmale eindeutig von epidemiologisch unbedeutenden Stämmen zu unterscheiden. Ideale Typisiermerkmale sollten deshalb innerhalb der Isolate des epidemischen Stammes stabil und innerhalb der gesamten Speziespopulation ausreichend polymorph (divers) sein. Diversität resultiert aus zufälligen, nicht letalen genetischen Ereignissen, die durch das Typisiersystem erkannt werden. Hierzu zählen z. B. neutrale Mutationen, die mittels multilocus-sequence-typing erfasst werden, oder Deletionen, Insertionen und Inversionen, die den Abstand zwischen Restriktionsendonuklease-Schnittstellen und damit die entsprechenden Spaltungsmuster beeinflussen. Die genetische Diversität zwischen Stämmen mit klinischer und epidemiologischer Bedeutung im Hinblick auf Pathogenität oder Antibiotika- resistenz als Selektionsfaktoren kann dann eingeschränkt sein, wenn eine bestimmte klonale Gruppe mit optimaler Adaptation an einen bestimmten Wirtsorganismus oder an ein bestimmtes Ökosystem weit verbreitet auftritt. Hier hat aber die Entwicklung sensitiver molekularer Techniken während der vergangenen 10 Jahre erheblich dazu beigetragen, Subpopulationen von evolutionär verwandten Erregergruppen (klonalen Gruppen) zu unterscheiden.

An die molekularen Methoden der Typisierung (Typisiersysteme s. [14, 15]) werden folgende Anforderungen gestellt:

D Die für die Typisierung verwendeten Merkmale sollten bei fast allen Isolaten der jeweiligen Erregerspezies nachweisbar sein, um eine hohe Typisierbarkeit ( 95\%) zu gewährleisten,

D die Ergebnisse der Typisierung müssen bei wiederholten, unabhängigen Versuchsansätzen im gleichen Laboratorium und zwischen verschiedenen Laboratorien seriell reproduzierbar sein,

D die Merkmale sollten im Verlauf eines Ausbruchs ausreichend stabil sein,

D ein Typisierverfahren muss innerhalb der Speziespopulation zur Unterscheidung einer ausreichenden „Typenanzahl“ führen, dabei sollte der Anteil des häufigsten „Typs“ $5 \%$ der Isolate einer Speziespopulation nicht übersteigen.

Molekulare Typisierung (Genotypisierung) bedeutet die Unterscheidung von Isolaten der gleichen Erregerspezies aufgrund von Unterschieden auf der Nukleinsäureebene. Sie wird sich notwendigerweise auf genomische Regionen mit dem erwünschten Maß an Variabilität konzentrieren oder mit geeigneten $\mathrm{Me}$ thoden genomische Strukturumbauten (Deletionen, Additionen, Inversionen) erfassen. Dabei sind verschiedene Ebenen der Unterscheidung erforderlich: Die Ebene mit der höchsten Empfindlichkeit muss ein Typisiermuster ergeben, das einen einzelnen Stamm und seine klonale Nachkommenschaft (z. B. im Falle eines Ausbruchs von Infektionen) von nicht verwandten Stämmen unterscheidet. Die zweite Ebene sollte eine Gruppe jeweils evolutionär ver- 
Tabelle 6

Liste der zu erfassenden Erreger mit Antibiotikaresistenzen gemäß Infektionsschutzgesetz $\S 23$ Abs. 1 S. 1

Erregerspezies

Zu erfassen ist die Resistenz (auch Einzelresistenzen) gegen folgende Substanzen, sofern im Rahmen der klinischmikrobiologischen Diagnostik getestet

\begin{tabular}{|c|c|}
\hline 1 Staphylococcus aureus & $\begin{array}{l}\text { Oxacillin, Vancomycin, Teicoplanin, Gentamicin, Chinolon Gr.IV } \\
\text { (z. B.Moxifloxacin), Quinupristin/Dalfopristin, Linezolid }\end{array}$ \\
\hline 2 Streptococcus pneumoniae & $\begin{array}{l}\text { Penicillin (Oxacillin } 1 \mu \mathrm{g} \text { ), Vancomycin, Cefotaxim, Erythromycin, } \\
\text { Chinolon Gr.IV (z. B.Moxifloxacin) }\end{array}$ \\
\hline $\begin{array}{l}3 \text { Enterococcus faecalis } \\
\text { Enterococcus faecium }\end{array}$ & $\begin{array}{l}\text { Vancomycin, Teicoplanin, Gentamicin und Streptomycin [beide, high } \\
\text { level“: Gentamicin }>500 \mathrm{mg} / \mathrm{l} ; \text { Streptomycin }>1.000 \mathrm{mg} / \mathrm{l} \text { (Mikrodil.) } \\
\text { bzw. } 2.000 \mathrm{mg} / \mathrm{l} \text { (Agardilution)], Linezolid } \\
\text { E. faecium: zusätzlich Quinupristin/Dalfopristin }\end{array}$ \\
\hline $\begin{array}{l}4 \text { Escherichia coli } \\
\text { Klebsiella spp. }\end{array}$ & $\begin{array}{l}\text { Imipenem/Meropenem, Chinolon Gr.II (z. B.Ciprofloxacin), Amikacin, } \\
\text { Ceftazidim, Piperacillin/Tazobactam, Cefotaxim oder analoge } \\
\text { Testsubstanz }^{\mathrm{a}} \text {, Cefoxitin }{ }^{\mathrm{b}}\end{array}$ \\
\hline $\begin{array}{l}5 \text { Enterobacter cloacae } \\
\text { Citrobacter spp. } \\
\text { Serratia marcescens }\end{array}$ & Imipenem/Meropenem, Chinolon Gr.II (z. B. Ciprofloxacin), Amikacin \\
\hline
\end{tabular}

aZur Bestätigung von ESBL:Cefpodixim+/-Clavulansäure, ${ }^{b}$ zur Bestätigung von Klasse C- $\beta$-Laktamasen.

Tabelle 7

Nachweis des ESBL-Charakters bei Enterobacteriaceae (ausführliche Übersicht bei [5])

\section{Kriterien für ESBL}

1.Plasmidkodierte $\beta$-Laktamasen

MHK für

Cefotaxim $1 \mathrm{mg} / \mathrm{l}$

Cetazidim $1 \mathrm{mg} / \mathrm{l}$

Cefpodoxim $4 \mathrm{mg} / \mathrm{l}$

Absenkung der Cefpodoxim-MHK durch Clavulansäure

um 3 Stufen (bzw. Hemmhofvergrößerung für Cepodoxim

um $5 \mathrm{~mm}$ )

2. Klasse C- $\beta$-Laktamasen (übertragbar, wenn Nachweis

in K.pneumoniae)

MHK für Cefoxitin mindestens $4 \mathrm{mg} / \mathrm{l}$, keine Absenkung

der Cefpodoxim-MHK bzw. keine Hemmhofvergrößerung

durch Clavulansäure

ESBL Extended Spectrum $\beta$-Laktamase; MHK minimale Hemmstoffkonzentration.

wandter Stämme voneinander unterscheiden. Eine dritte Ebene beschreibt Erregerstämme, die zunächst unabhängig von einer genotypischen Verwandtschaft in Verbindung mit bestimmten Erkrankungen oder klinischen Situationen stehen. Tabelle 8 fasst die wichtigsten Methoden der molekularen Erregertypisierung zusammen.

\section{Interpretation}

Resistenz gegen alle Penicilline

und Cephalosporine

\section{Anwendung der Typisierung im Rahmen von Studien zur Resistenzentwicklung}

Außer zur Aufklärung von Infektketten mit resistenten Erregern (z. B. MRSA) ist die Typisierung für die Klärung der Ursachen/Mechanismen, die einer Resistenzausbreitung zugrunde liegen, uner- lässlich. Diesbezüglich gibt es 3 verschiedene Möglichkeiten:

1.Möglichkeit. Die beobachtete Zunahme einer Antibiotikaresistenz beruht auf einer Verbreitung von Resistenzgenen bzw. von Resistenzgenclustern zwischen einer Vielzahl unterschiedlicher Bakterienstämme im Sinne eines Resistenzgen- oder eines Resistenzplasmid-Hospitalismus.

Um zu klären, ob dieser Mechanismus für die Zunahme einer Resistenz verantwortlich ist, ist zusätzlich zur Typisierung des genomischen Hintergrundes auch eine Charakterisierung der Resistenzgene bzw. Resistenzgencluster sinnvoll. Bei Enterobacteriaceae kann der ESBL-Phänotyp von einer Vielzahl verschiedener Mutanten der weit verbreiteten $\beta$-Laktamasen des TEM- und des SHV-Typs ausgehen, die durch PCR und eine nachfolgende Sequenzierung voneinander unterschieden werden [25]. Glykopeptidresistente Enterokokken in Europa besitzen überwiegend das vanAGen. Aufgrund von Insertionen und Deletionen sowie Punktmutationen im vanA-Gencluster (Tn1546 like elements) ist eine Unterscheidung einzelner „Subtypen“ gut möglich ([26], Überblick zur Anwendung im Rahmen ökologischer Studien bei [27]). Das die Methicillinresistenz bei Staphylokokken kodierende $m e c \mathrm{~A}-\mathrm{Gen}$ ist in größere Elemente (staphylococcal cassette chromosome mec, SCCmec) integriert, die bis $\mathrm{zu} 60 \mathrm{~kb}$ groß sein können. Bisher wurden aus Sequenzierungen die Typen I, II, III, IVa und IVb abgeleitet [28]. Unterschiedliche MRSA-Epidemiestämme und community acquired MRSA tragen verschiedene SCCmec-Elemente.

Der hier vorgestellte Mechanismus der Resistenzausbreitung, d. h. die Verbreitung von Resistenzgenen bzw. von Genclustern zwischen einer Vielzahl von Stämmen, gilt hauptsächlich für die $\beta$ Laktamasen mit erweitertem Wirkungsspektrum (ESBL). Beispielhaft dafür sind Ergebnisse aus einem spanischen Krankenhaus. Hier traten ESBL der Typen TEM-4, SHV-2 und CTX 10 in genotypisch verschiedenen Stämmen der Spezies E. coli und K. pneumoniae auf [29].

2.Möglichkeit. Die Zunahme einer Resistenz beruht auf der Verbreitung eines bestimmten Stammes (einer bestimm- 


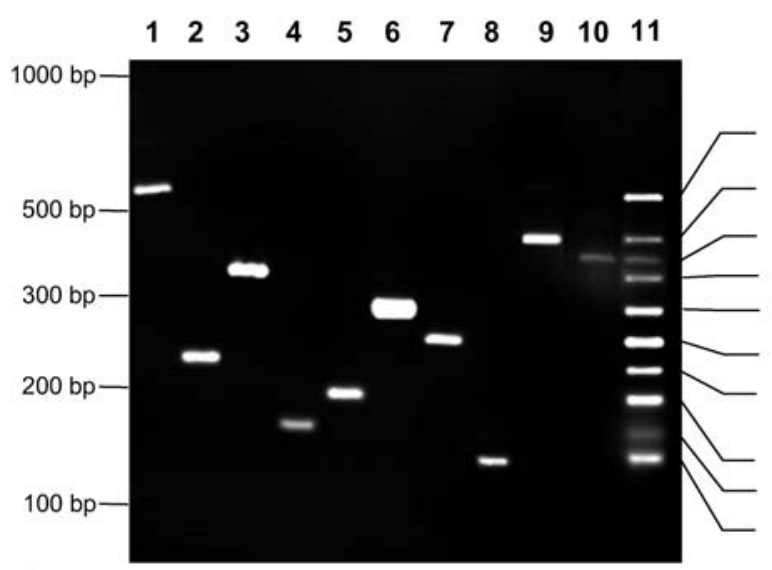

a $\begin{array}{llllllllllll}11 & 10 & 9 & 8 & 7 & 6 & 5 & 4 & 3 & 2 & 1 & M\end{array}$

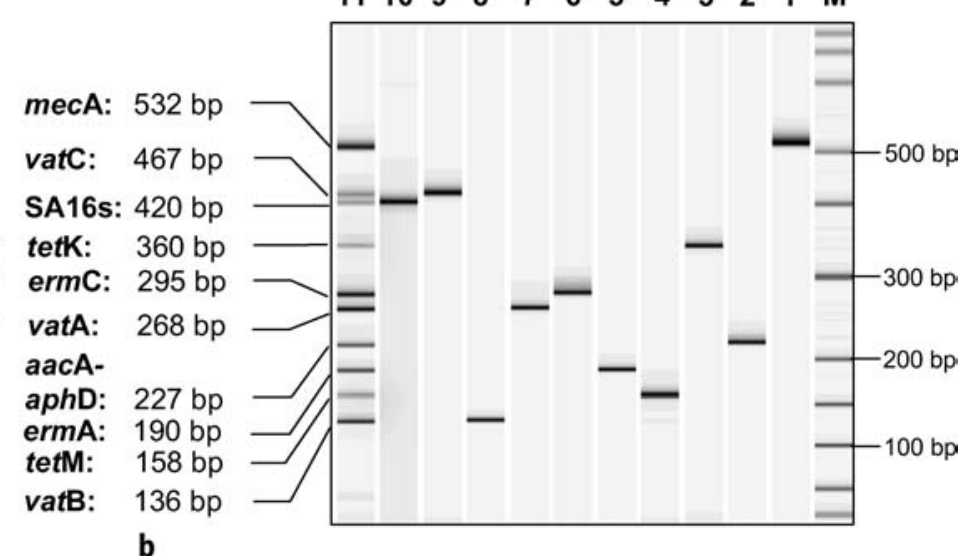

b

Abb. 3a, b $\Delta$ Einzel-PCR-Produkte (1-10) und ein Multiplex-PCR-Produkt (11) für 9 klinisch relevante Antibiotikaresistenzgene und eine Amplifikationskontrolle aus der 16 s rDNA. a Darstellung mittels Gelelektrophorese und Ethidiumbromidfärbung. b Darstellung mittels Kapillarelektrophorese
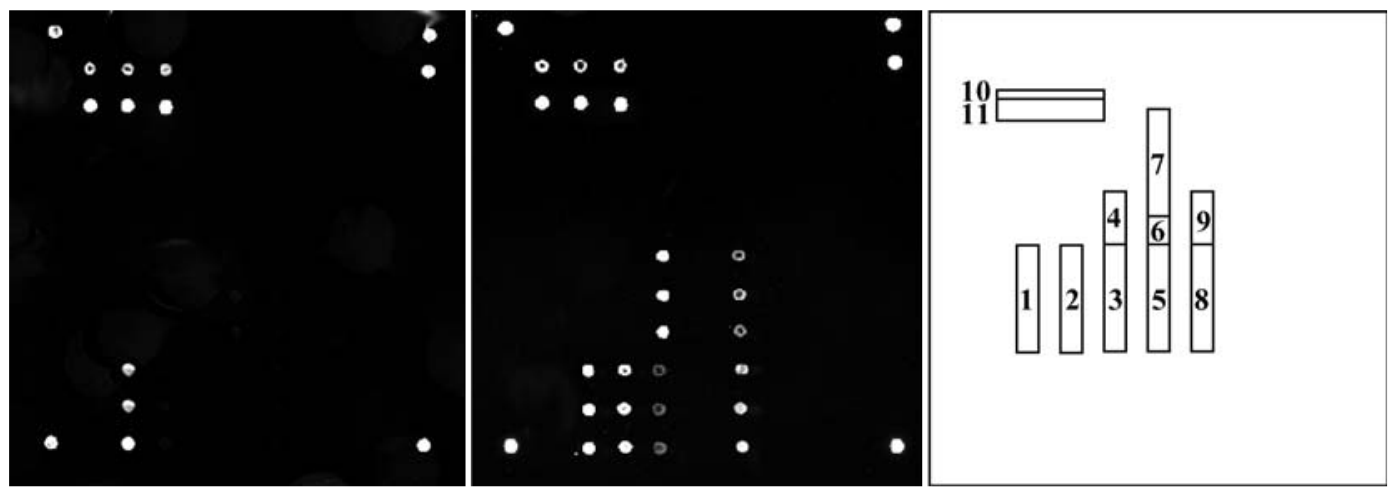

Abb. $4 \Delta$ Microarray-Hybridisierungsmuster für 2 verschiedene Methicillin-resistente S. aureus (MRSA). a Isolat mit schmalem Resistenzspektrum (mec $A$ ). $b$ Isolat mit breitem Resistenzspektrum (mecA, aacA-aphD, tetK, tetM, ermA, ermC), c Legende; 1: mecA, 2: aacA-aphD, 3: tetK, 4: tetM, 5: vatA, 6: vatB, 7: vatC, 8: ermA, 9: ermC, 10: Hybridisierungskontrolle, 11: Amplifikationskontrolle

ten Subpopulation der Erregerspezies), der an verschiedenen Orten und zu verschiedenen Zeiten Resistenzgene oder zur Resistenz führende Mutationen erwarb.

Ein Beispiel für diesen Mechanismus der Resistenzausbreitung ist die Verbreitung der C1-Population von E. faecium in europäischen Krankenhäusern. Seine Existenz wurde erst durch die Anwendung der AFLP- und MLST-Verfahren (Tabelle 8) offensichtlich. Der Erwerb des esp-Gens (das Gen kodiert für ein Protein der intrazellulären Adhäsion bei Biofilmbildung, vermutlich auch für Adhäsion) sowie der van-Gencluster und der sat-Gene (für Streptogramin-A-Resistenz) erfolgten und erfolgen weiterhin offenbar unabhängig voneinander [30]. Der Nachweis von van $\mathrm{A}-\mathrm{Gen}$ tragenden E. faecium mit ähnlichen Makrorestriktionsmustern in einem Krankenhaus über längere Zeitabstände bzw. überregional in verschiedenen Krankenhäusern kann deshalb nicht ohne eine vertiefende Charakterisierung als klonale Ausbreitung interpretiert werden (Beispiele dafür bei $[31,32])$.

3. Möglichkeit. Die Zunahme einer Resistenz beruht auf der klonalen Ausbreitung eines resistenten (multiresistenten) Epidemiestammes.

Dieser Mechanismus ist für den weltweit beobachteten Anstieg von MRSA verantwortlich. Dieser Anstieg geht auf die klonale Verbreitung (in Krankenhäusern, zwischen Krankenhäusern, über Landesgrenzen und auch interkontinental) von mehreren Epidemiestämmen zurück, die durch SmaIMakrorestriktionsmuster [33], MLSTTypisierung und Charakterisierung der
SCCmec-Elemente charakterisiert sind $[34,35]$. Aber auch bei gramnegativen Erregern von Hospitalinfektionen wurde über eine weit reichende klonale Ausbreitung bestimmter resistenter Stämme berichtet (Zusammenfassung bei [21]). Bemerkenswert ist die Ausbreitung eines S.-enterica-Serovar-Typhimurium-Stammes, der die ESBL CTXM-4 bildet, in Russland, Ungarn und Griechenland [36].

\section{Ergänzung der Typisierung durch die Bestimmung von Merkmalen mit Bedeutung für die Diagnostik nosokomia- ler Infektionen}

Die bakteriellen Erreger nosokomialer Infektionen sind an sich Bestandteil der normalen Besiedlungsflora des Menschen sowie der Umwelt. Dabei können einzelne Populationsanteile der Besiedlungsflora besondere Pathogenitätsmerkmale aufweisen, die für die Bestätigung der klinischen Diagnose von Bedeutung sind und zudem auch einen 


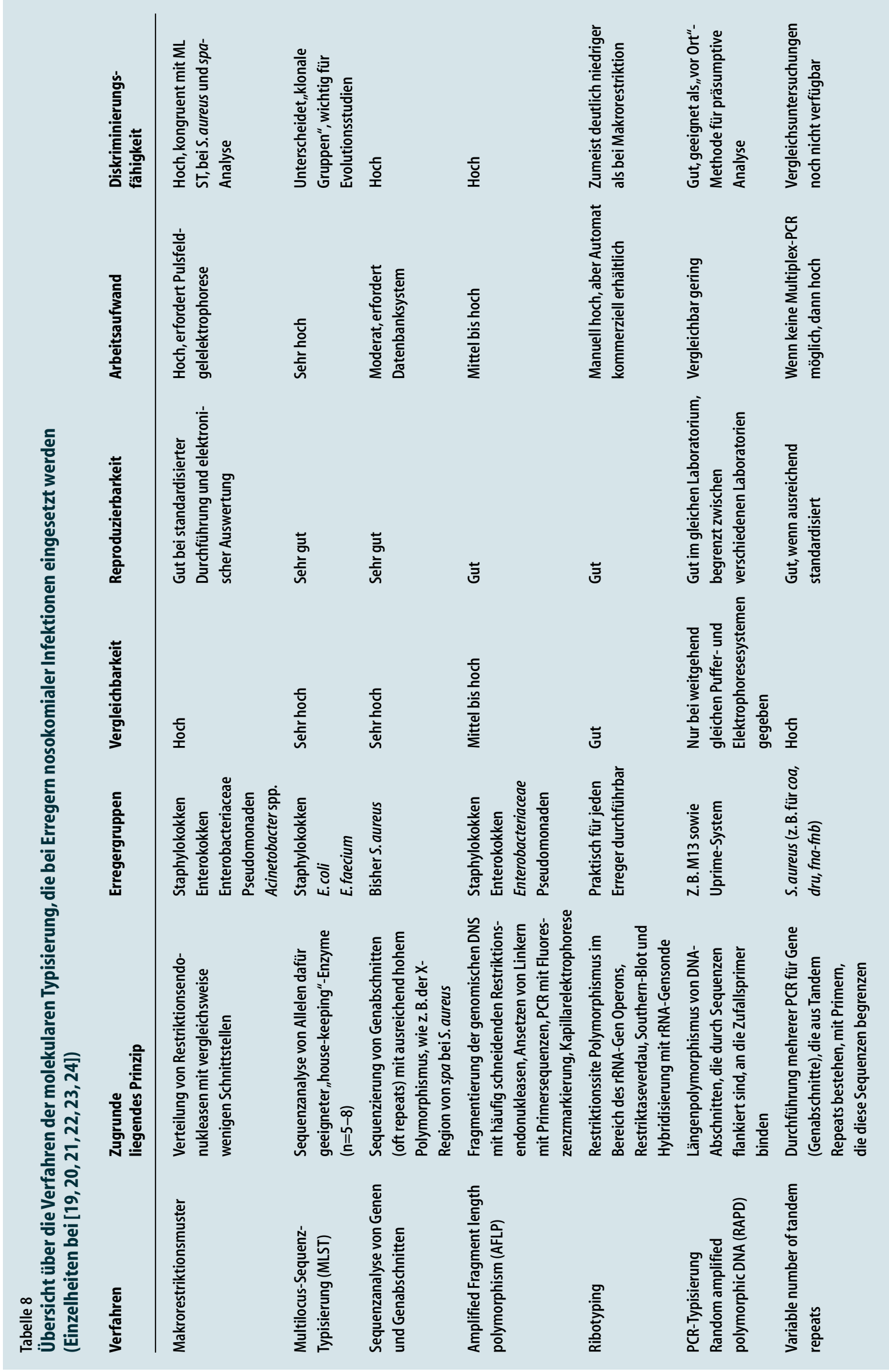


Tabelle 9

Beispiele für den Nachweis von Erregereigenschaften zur Bestätigung der klinischen Diagnose und mit prädiktivem Wert für den Krankheitsverlauf

\begin{tabular}{|c|c|c|c|}
\hline Erreger & Art der Infektion & Genetische Determinante & $\begin{array}{l}\text { Bisher etablierte } \\
\text { Nachweise }\end{array}$ \\
\hline \multirow[t]{3}{*}{$\begin{array}{l}\text { S. aureus } \\
{[37,38,39} \\
40,41,42]\end{array}$} & Toxic Shock Syndrom & $\begin{array}{l}\text { Superantigene Toxic Shock } \\
\text { Syndrom Toxin (tst), selten } \\
\text { Enterotoxine B und C (seb, sec) }\end{array}$ & PCR \\
\hline & $\begin{array}{l}\text { Exfoliative Dermatitis } \\
\text { (SSSS) }\end{array}$ & $\begin{array}{l}\text { Exfoliative Toxine A und B } \\
\text { (eta, etb) }\end{array}$ & PCR \\
\hline & $\begin{array}{l}\text { Tief gehende Hautinfek- } \\
\text { tionen, nekrotisierende } \\
\text { Pneumonien }\end{array}$ & $\begin{array}{l}\text { Panton-Valentin-Leukozidin } \\
\text { (lukS-IukF) }\end{array}$ & PCR \\
\hline $\begin{array}{l}\text { E. faecium } \\
\text { [43] }\end{array}$ & Systemische Infektionen & $\begin{array}{l}\text { Intrazelluläres Adhäsin, Biofilm- } \\
\text { bildung, enterococcal surface } \\
\text { protein (esp) }\end{array}$ & PCR \\
\hline $\begin{array}{l}\text { E. coli } \\
{[44,45,46,} \\
47,48,49]\end{array}$ & $\begin{array}{l}\text { Sepsis, Meningitis, } \\
\text { Urosepsis, hämolytisch- } \\
\text { urämisches Syndrom (HUS) }\end{array}$ & $\begin{array}{l}\text { Nekrotisierendes Zytotoxin (cnf-1), } \\
\text { Pyelonephritis-Adhäsin (Pili, papC), } \\
\alpha \text {-Hämolysin (hly), Aerobactin (aero), } \\
\text { Non-Fimbrien-Adhäsin (afa), } \\
\text { Shiga-Toxin (stx),Intimin (eae) }\end{array}$ & $\begin{array}{l}\text { Dot-blot } \\
\text { und PCR-probes, } \\
\text { Array-Technologie }\end{array}$ \\
\hline
\end{tabular}

prädiktiven Wert besitzen (Tabelle 9). Dies wird bisher vor allem für Staphylococcus aureus praktisch angewandt. Bei E. coli ist es von besonderem Interesse, Isolate mit hoher pathogener Potenz im Hinblick auf Sepsis, Urosepsis und Meningitis rechtzeitig zu erkennen. Hier wirken mehrere Pathogenitätsmechanismen zusammen, die sich vor allem bei der Subpopulation B2 finden (Tabelle 9). In epidemiologischen Studien wurden diese virulenzassoziierten Gene bisher vergleichsweise aufwändig mittels Dot-blot-Analyse bestimmt. Aussichtsreich ist hier die Einführung von Mikroarrays [47].

Grundsätzlich ist es aber nicht möglich, innerhalb einer Spezies harmlose Besiedler von Stämmen mit hoher pathogener Potenz zu unterscheiden. Nosokomiale Infektionen betreffen häufig Patienten, die aufgrund von Grunderkrankungen, von Immunschwächen und von unausweichlichen Behandlungsverfahren (z. B. Langzeitbeatmung) für Infektionen prädisponiert sind. Ausgangspunkt einer Infektion kann dann bereits die Anwesenheit einer „Besiedlers“ zur falschen Zeit am falschen Ort sein.

\section{Literatur}

1. Davies J (1997) Origins, acquisition and dissemination of antibiotic resistance determinants. In:Levy SB (ed) Antibiotic resistance: origins, evolution, selection and spread. John Wiley \& Sons, Chichester New York, pp 1-8

2. Witte W (1999) Antibiotic resistance in grampositive bacteria: epidemiological aspects.J Antimicrob Chemother 44 [Suppl A]:1-9

3. Shaw KJ, Rather PN, Hare RS, Miller GH (1993) Molecular genetics of aminoglycoside resistance genes and familiar relationships of aminoglycoside modifying enzymes. Microbiol Rev 57:138-163

4. Miller HG, Sabatelli JF, Hare RS et al. (1997) The most frequent aminoglycoside resistance mechanism - changes with time and geographic area: a reflection of aminoglycoside usage patterns? Clin Infect Dis 24:46-62

5. Witte W, Mielke M (2003) $\beta$-Laktamasen mit breitem Wirkungsspektrum. Bundesgesundheitsbl Gesundheitsforsch Gesundheitsschutz 46:881-890

6. DIN-Standard 58940; Chemotherapeutische Untersuchungsmethoden

7. NCCLS-Standard M100-S12. Performance Standards for Antimicrobial Susceptibility Testing; Twelfth Informational Supplement, January 2002

8. Cuny C, Pasemann B, Witte W (1999) Detection of oxacillin resistance in Staphylococcus aureus by screening tests.

Eur J Clin Microbiol Infect Dis 11:834-836
9. Van Leeuwen W, van Pelt C, Luijendijk A et al. (1999) Rapid detection of methicillin resistance in Staphylococcus aureus isolates by the MRSA-screen latex agglutination test. $\mathrm{J}$ Clin Microbiol 37:3029-3030

10. Cuny C, Salmenlinna S, Witte W (2001) Evaluation of a reverse hybridization blot test for detection of oxacillin-resistant Staphylococcus aureus. Eur J Clin Microbiol Infect Dis 20:906-907

11.www.bag-germany@com

12. Strommenger B, Kettlitz C, Werner G, Witte W (2003) Multiplex PCR assay for simultaneous detection of nine clinically relevant resistance genes in Staphylococcus aureus.J Clin Microbiol 41:4089-4094

13. Perez-Perez FJ, Hansen ND (2002) Detection of plasmid-mediated AmpC beta-lactamase genes in clinical isolates using multiplex PCR. J Clin Microbiol 40:2153-2162

14. Call DR, Bakko MK, Krug MJ, Roberts MC (2003) Identifying antimicrobial resistance genes with DNA microarrays. Antimicrob. Agents Chemother 47:3290-3295

15. Volokhov D, Chizhikov V, Chumakov K, Rasooly A (2003) Microarray analysis of erythromycin resistance determinants. J Appl Microbiol 95:787-798

16. Reischl U, Linde HJ, Metz M et al. (2000) Rapid identification of methicillin-resistant Staphylococcus aureus and simultaneous species confirmation using real-time fluorescence $P C R$. $J$ Clin Microbiol 38:2429-2433

17. Tan TY, Corden S, Barnes R, Cookson B (2001) Rapid identification of methicillin-resistant Staphylococcus aureus from positive blood cultures by real-time fluorescence $P C R$. J Clin Microbiol 39:4529-4531

18. Struelens M (1996) Consensus guidelines for appropriate use and evaluation of microbial epidemiologic typing systems. Clin Microbiol Infect 2:2-11

19. Goering RV (1998) The molecular epidemiology of nosocomial infection. An overview of principles, application, and interpretation. In: Specter $G$ (ed) Rapid detection of infectious agents. Plenum Press, New York

20. Witte W (1999) Diagnostics, typing \& taxonomy. In: Fischetti VA, Fenetti J, Novick RP (eds) Grampositive pathogens. ASM, Washington, DC, pp 309-316

21. Savekoul P, Aarts HJ, de Haas J et al. (1999) Amplified fragment length polymorphism analysis: the state of the art.J Clin Microbiol 37:3083-3091

22. Enright MC, Spratt BG (1999) Multilocus sequence typing. Trends Microbiol 7:482-487

23. Sabat A, Kryszton-Russjan J, Strzalka W et al. (2003) New method for typing Staphylococcus aureus strains: multiple-locus variable tandem repeat analysis of polymorphism and genetic relationships of clinical isolates.J Clin Microbiol 41:1801-1804 


\section{Leitthema:Krankenhaushygiene}

24. Harmsen D, Claus H, Witte W et al. (2003) Typing of methicillin-resistant Staphylococcus aureus in a university hospital setting using a novel software for spa repeat determination and database management.J Clin Microbiol 41 (in press)

25. Gniadkowski M (2001) Evolution and epidemiology of extended spectrum $\beta$-lactamases (ESBL's) and ESBL microorganisms. Clin Microbiol Infect 7:597-608

26. Willems R, Top J, Van den Braak N et al. (1999) Molecular diversity and evolutionary relationships of Tn1546-like elements in enterococci from humans and animals. Antimicrob Agents Chemother 43:483-491

27. Witte W, Klare I, Werner G (2002) Molecular ecological studies on spread of antibiotic resistance genes. Anim Biotechnol 13:57-70

28. Ma XX, Ito T,Tiensasitorn C et al. (2002) Novel type of staphylococcal cassette chromosome mec identified in community-acquired methicillin-resistant Staphylococcus aureus. Antimicrob Agents Chemother 46:1147-1152

29. Coque TM, Oliver A, Perez-Diaz JC et al. (2002) Genes encoding TCM-4, SHV2, and CTX-M-10 extended-spectrum beta lactamases are carried by multiple Klebsiella pneumoniae clones in a single hospital (Madrid, 1988-2000). Antimicrob Agents Chemother 46:500-510

30. Homann W, Tribe D, Poznanski S et al. (2002) Multilocus sequence typing scheme for Enterococcus faecium.J Clin Microbiol 40:1963-1971

31. Oancea C, Werner G, Klare I, Witte W (2003) Transfer of esp between enterococcal strains co-selected by antibiotic resistance gene transfer.6th IMMEM, 27.-30.08.2003, Les Diablerets, Switzerland, Abstract 03-A-87-ASM
32. Werner G, Klare I, Spencker FB, Witte W (2003) Intra-hospital dissemination of quinupristin/dalfopristin and vancomycin-resistant Enterococcus faecium in paediatric ward of a German hospital.J Antimicrob Chemother 52:113-115

33. Witte W, Braulke C, Cuny C et al. (2002) Changing pattern of antibiotic resistance in methicillin-resistant Staphylococcus aureus from German hospitals. Infect Control Hosp Epidemiol 22:683-686

34. Enright ML, Robinson DA, Feil EJ et al. (2002) The evolutionary history of methicillin-resistant Staphylococcus aureus (MRSA). Proc Nat Acad Sci 28:7687-7692

35. Branger C, Gardye C, Galdbart J0 et al. (2003) Genetic relationship between methicillinresistant Staphylococcus aureus strains from France and from international sources: delineation of genomic groups.J Clin Microbiol 41:2946-2951

36. Tassios PT, Gazouli M, Tzelepi E (1999) Spread of a Salmonella typhimurium clone resistant to expanded-spectrum cephalosporins in three European countries.J Clin Microbiol 37:3774-3777

37. Dinges $M, O$ rwin PM, Schlievert P (2000) Exotoxins of Staphylococcus aureus. Clin Microbiol Rev 13:16-34

38. Johnson WM, Tyler SD, Ewan EP et al. (1991) Detection of genes for enterotoxins, exfoliative toxins, and toxic shock syndrome toxin 1 in Staphylococcus aureus by the polymerase chain reaction. J Clin Microbiol 29:426-430

39. Becker K, Roth R, Peters G (1998) Rapid and specific detection of toxigenic Staphylococcus aureus: use of two multiplex PCR immunoassays for amplification and hybridization of staphylococcal enterotoxin genes, exfoliative toxin genes and toxic shock syndrome toxin-1 gene.J Clin Microbiol 36:2548-2553

40. Becker C, Friedrich AW, Lubritz G et al. (2003) Prevalence of genes encoding pyrogenic toxin superantigens and exfoliative toxins among strains of nasal specimens.J Clin Microbiol 41:1434-1439

41. Lina $G$, Piemont $Y$, Godail-Gamot $F$ et al. (1999) Involvement of Panton-Valentine Leukocidineproducing staphylococcus aureus in primary skin infections and pneumoniae. Clin Infect Dis 29:1128-1132

42. Cuny C, Braulke C, Strommenger B et al. (2004) Leukocidine determinants in Staphylococcus aureus of different clinical origin. Int J Med Microbiol 293: im Druck

43. Willems R, Homann W, Top J et al. (2001) Variant esp as a marker of a distinct genetic lineage of vancomycin-resistant Enterococcus faecium spreading in hospitals. Lancet 357:853-855

44. Hilali F, Ruimy R, Saulnier P et al. (2000) Prevalence of virulence genes and clonality in Escherichia coli strains that cause bacteremia in cancer patients. Infect. Immunity 68:3983-3989
45. Johnson JR, Oswald E, O'Brian T et al. (2002) Phylogenetic distribution of virulence associated genes among Escherichia coli isolates associated with neonatal meningitis in the Netherlands.J Infect Dis 185:774-784

46. Bingen-Bidois $\mathrm{M}$, Chlermont $\mathrm{O}$, Bonacorsi $\mathrm{S}$ et al. (2002) Phylogenetic analysis and prevalence of urosepsis strains of Escherichia coli bearing pathogenicity island-like domains. Infect Immun 70:3216-3226

47. Dobrindt U, Agere F, Janka A et al. (2003) Analysis of genomic plasticity in pathogenic and commensal Escherichia coli isolates by use of DNA arrays.J Bacteriol 185:1831-1840

48. Fratamico PM, Sackitey SV, Wiedmann M, Deng MY (1995) Detection of Escherichia coli 0175:H7 by multiplex PCR.J Clin Microbiol 33:2188-2191

49. Friedrich AW, Bielaszewska $M$, Zhang WC et al. (2002) Escherichia coli harbouring Shiga toxin 2 gene variants: frequency and association with clinical symptoms.J Infect Dis 185:74-84 